\title{
LUNG BIOPSY: IS IT NECESSARY?
}

Roy T. Temes, MD

Nancy E. Joste, $\mathrm{MD}^{\mathrm{b}}$

Clifford R. Qualls, $\mathrm{PhD}^{\mathrm{c}}$

Nechol L. Allen, MS

Richard E. Crowell, MD ${ }^{\mathrm{d}}$

Hector A. Dox, MD

Jorge A. Wernly, MD
Objective: Lung biopsy is associated with substantial mortality rates. We reviewed our experience with this operation, primarily in patients with immunocompetence, to determine whether the results justify the continued performance of this procedure. Methods: We conducted a retrospective review of all diagnostic lung biopsies performed at 3 university-affiliated hospitals between July 1, 1992, and December 31, 1998. Results: There were 75 patients: 25 patients were treated electively, 17 were treated on an urgent basis, 27 patients on an emergency basis, and the urgency was unclear in 6 patients. Significant beneficial therapeutic changes were made in 15 of 25 elective procedures $(60 \%)$, in 16 of 17 urgent procedures $(94 \%)$, and in 11 of 27 emergency procedures $(41 \%$; $P=.001)$. Significant beneficial therapeutic changes consisted of immunosuppression in 13 of $15(87 \%)$ patients treated on an elective basis, in 9 of $16(56 \%)$ treated on an urgent basis, and in 9 of $11(82 \%)$ treated on an emergency basis in whom therapy was altered $(P=.14)$. Operative death was 0 of 25 for elective operations $(0 \%), 3$ of 17 for urgent operations (18\%), and 14 of 26 for emergency operations (54\%). Multivariable analysis of operative death showed urgency to be the only significant predictor of death $(P=\mathbf{. 0 0 2})$. Conclusions: In patients with immunocompetence, elective and urgent lung biopsies have acceptable operative mortality rates and frequently result in important beneficial therapeutic changes. Consequently biopsies are appropriate in these patients. Emergency biopsies are associated with high operative mortality rates and rarely result in a therapeutic change other than immunosuppression. These patients should not undergo lung biopsy if they are in stable condition and should be treated empirically with immunosuppression without operation if their condition is deteriorating. ( $J$ Thorac Cardiovasc Surg 1999;118:1097-100)
L ung biopsy is eventually performed in 1 of 3 patients with interstitial lung disease of unknown cause. However, it is associated with a substantial mortality rate. We reviewed our experience with this procedure to determine whether the mortality rate is outweighed by vital therapeutic interventions and a survival benefit.

From the University of New Mexico Health Sciences Center, Department of Surgery, ${ }^{\mathrm{a}}$ Department of Pathology, ${ }^{\mathrm{b}}$ Department of Mathematics and Statistics, ${ }^{\mathrm{c}}$ and Department of Medicine, ${ }^{\mathrm{d}}$ Albuquerque, NM.

Received for publication April 6, 1999; revisions requested June 29, 1999; revisions received July 20, 1999; accepted for publication Aug 12, 1999.

Address for reprints: R. Thomas Temes, MD, University of New Mexico, Department of Surgery, 2211 Lomas Blvd NE, Albuquerque, NM 87131 (E-mail: Ttemes@salud.unm.edu).

Copyright (C) 1999 by Mosby, Inc.

$0022-5223 / 99 \$ 8.00+0 \quad \mathbf{1 2 / 1 / 1 0 2 4 8 2}$

\section{Material and methods}

All diagnostic surgical lung biopsies performed for diffuse nodular or interstitial pulmonary disease at 3 university-affiliated hospitals between July 1, 1992, and December 31, 1998, were retrospectively reviewed. Patients with solitary nodules or other focal pulmonary processes were excluded.

Elective operations were defined as scheduled admissions to the hospital exclusively for the operative procedures. Urgent operations were defined as biopsies performed during hospitalization for respiratory failure in patients not requiring $100 \%$ non-rebreather face mask oxygen supplementation or mechanical ventilation. Emergency biopsies were defined as biopsies performed during hospitalization for respiratory failure in patients receiving $100 \%$ non-rebreather face mask oxygen supplementation or mechanical ventilation.

Significant beneficial therapeutic changes were defined as new treatments initiated as a result of the lung biopsy. Withdrawal of medications, withdrawal of other therapies, or withdrawal of life support were not considered significant 
beneficial therapeutic changes. Operative death was defined as death during the same hospitalization or within 30 days of the operation.

Results were analyzed by age, sex, urgency, technique, biopsy site, diagnostic yield, pathologic results, culture results, whether a significant beneficial therapeutic change was made, type of therapy change, and operative mortality rate.

Comparisons between groups were performed with the Fisher exact test for univariable analysis. Multivariable stepwise logistic regression was performed for operative death of all candidate variables. These variables included age, sex, urgency, surgical technique, significant beneficial therapeutic change, and type of therapy change. Histologic diagnosis was established by review of pathologic reports.

\section{Results}

Twenty-six patients were female, and 49 patients were male. The mean age was 56 years (range 7-85 years). There were 75 biopsies performed. The numbers of biopsies performed per year were 3 ( 0 elective, 1 urgent, 1 emergency, 1 urgency unknown) in 1992, 13 (3 elective, 2 urgent, 5 emergency, 3 urgency unknown) in 1993, 11 (5 elective, 2 urgent, 2 emergency, 2 urgency unknown) in 1994, 10 (5 elective, 1 urgent, 4 emergency) in 1995, 19 (6 elective, 4 urgent, 9 emergency) in 1996, 11 (4 elective, 4 urgent, 3 emergency) in 1997, and 8 ( 2 elective, 3 urgent, 3 emergency) in $1998(P=.9)$.

Open procedures were performed in 0 of 25 of elective operations $(0 \%)$, in 4 of 17 urgent operations (24\%), and in 24 of 27 emergency operations (89\%; $P$ $<.001)$. Video thoracoscopic procedures were performed whenever possible. Open procedures were chosen for critically ill patients to minimize operative time, avoid reintubation with a double lumen tube, and abstain from single lung ventilation.

The site of the biopsy was the lingula in 42 cases, left upper lobe otherwise not specified in 1 case, left lower lobe in 27 cases, right upper lobe in 9 cases, right middle lobe in 9 cases, right lower lobe in 16 cases, and unspecified sites in 6 patients. There was no significant difference in the location of the biopsies between the urgency groups $(P=.24)$. Thirty-four patients underwent biopsy of a single location; 31 patients had biopsies obtained from 2 lobes; and 4 patients had biopsies obtained from 3 lobes.

A diagnosis was achieved in $100 \%$ of patients. Usual interstitial pneumonia, bronchiolitis, diffuse alveolar damage, honeycomb lung, and pneumonia were the most common diagnoses (Table I). Diffuse alveolar damage was significantly more common in patients requiring emergency treatment; bronchiolitis was significantly more common in the group treated elective- ly, and granulomatous disease (infectious and noninfectious) was significantly more common in those receiving urgent treatment. Otherwise, there were no significant differences in histologic diagnosis between urgency groups.

Aerobic and anaerobic cultures results were available for 64 patients. Only 2 of 64 cultures (3\%) were positive for organisms: Propionibacterium acnes in 1 case and coagulase-negative Staphylococcus in the other case. Both were thought to be contaminants, and the results were disregarded. Tuberculosis culture results were available for 58 patients. None of the results were positive for tuberculosis, and none of the results were useful clinically. Fungal culture results were available for 57 patients. Only 4 of 57 cultures (7\%) were positive for fungal organisms: Candida was identified in 2 patients, Penicillium and Geotrichum were identified in 1 patient, and Histoplasma was identified in 1 patient. All but the Histoplasma were thought to be contaminants and not useful clinically. Although the Histoplasma represented true infection, it had already been diagnosed on the histologic specimen. Viral cultures were not routinely sent. The diagnosis of viral infections were most commonly made by findings on histologic sections.

Significant beneficial therapeutic changes were made in 15 of 25 elective biopsies (60\%), in 16 of 17 urgent biopsies (94\%), in 11 of 27 emergency biopsies (41\%), and in 42 of 69 biopsies $(61 \%)$ overall $(P=.001$; the changes made for 6 patients are unknown). Significant beneficial therapeutic changes in patients undergoing elective biopsy consisted of treatment with steroids, azathioprine, and/or cyclophosphamide in 13 of 15 patients (87\%) and chemotherapy in 2 of 15 patients (13\%). Significant beneficial therapeutic changes in patients undergoing urgent biopsy consisted of treatment with steroids, azathioprine, or cyclophosphamide (or a combination) in 9 of 16 patients (82\%), antiviral therapy in 1 of 16 patients (6\%), antifungal therapy in 2 of 16 patients (13\%), antibacterial antibiotics with steroids in 1 of 16 patients (6\%), chemotherapy in 1 of 16 patients $(6 \%)$, and heparin with or without steroids in 2 of 16 patients (13\%). Significant beneficial therapeutic changes in patients undergoing emergency biopsy consisted of treatment with steroids, azathioprine, or cyclophosphamide (or a combination) in 9 of 11 patients $(82 \%)$, antiviral therapy in 1 of 11 patients (9\%), and heparin with steroids in 1 of 11 patients $(9 \%)$. There were no significant differences in the usage of immunosuppression among patients having therapy changes within the 3 urgency groups $(P=.14)$. No patient died of surgical complications; however, 
Table I. Histologic diagnosis in 66 patients*

\begin{tabular}{lccccc}
\hline Diagnosis & Elective & Urgent & Emergency & Total & P value \\
\hline DAD & 0 & 0 & 11 & 11 & $<.001$ \\
Bronchiolitis & 8 & 3 & 1 & 12 & .03 \\
Granulomatous & 1 & 4 & 0 & 5 & .01 \\
UIP & 15 & 7 & 10 & 32 & .4 \\
DIP & 1 & 0 & 0 & 1 & 1.0 \\
Honeycomb & 4 & 4 & 2 & 10 & .4 \\
Emboli/thrombi & 0 & 2 & 3 & 5 & .17 \\
Vasculitis & 0 & 0 & 3 & 3 & .11 \\
Pneumonia & 2 & 2 & 6 & 10 & .3 \\
Malignancy & 2 & 2 & 0 & 4 & .18 \\
Hemorrhage & 0 & 1 & 2 & 3 & .4
\end{tabular}

$\overline{D A D}$, Diffuse alveolar damage; UIP, usual interstitial pneumonia; $D I P$, diffuse interstitial pneumonia.

*Six patients in whom the urgency was unknown are not included; more than 1 diagnosis was made in some patients.

many of the deaths occurred when life support was withdrawn after the diagnosis of irreversible end-stage pulmonary disease was established. Overall operative death occurred in 20 of 73 patients $(27 \%$; 2 patients unknown). There were no operative deaths in the elective group, $3(18 \%)$ operative deaths among 17 patients receiving urgent treatment, and $14(54 \%)$ operative deaths among 26 patients requiring emergency treatment. With the use of univariable analysis, operative death was strongly associated with urgency $(P<.001)$ and the use of open surgical technique $(P<.001$; Table II). With multivariable logistic regression analysis, only urgency remained as significant predictor of operative death $(P=.002)$.

\section{Discussion}

Previously reported results of lung biopsy depend significantly on the immunologic status of the patient. Because most of our patients were immunocompetent, we analyzed our results on the basis of the urgency of the procedure rather than the immunologic status. Our population was fairly evenly divided with $33 \%$ of patients undergoing elective biopsies, $23 \%$ undergoing urgent biopsies, and 36\% undergoing emergency procedures. Because of lower diagnostic yields, it was rare for patients to have undergone transbronchial lung biopsy before referral for surgical lung biopsy. Consequently, we did not review the results of transbronchial lung biopsies.

The number of biopsies, size of biopsies, blood loss, operative time, and diagnostic yield are equivalent for open and thoracoscopic techniques. However, duration of chest tube drainage, total days of hospitalization, and total cost are decreased by use of the thoracoscopic
Table II. Analysis of operative death by possible risk factors

\begin{tabular}{lcc}
\hline Risk factor & $\begin{array}{c}\text { Univariable } \\
\text { models } \\
\text { (P value) }\end{array}$ & $\begin{array}{c}\text { Multivariable } \\
\text { models } \\
\text { (P value) }\end{array}$ \\
\hline Urgency & $<.001$ & .002 \\
Open vs scope & $<.001$ & .17 \\
Male vs female & .6 & .7 \\
Aged > 70 y & .5 & .8 \\
Therapy change vs none & .6 & .3 \\
Immunosuppression vs none & .2 & .9 \\
\hline
\end{tabular}

technique. ${ }^{1,2}$ Therefore our preference is to use thoracoscopic procedures for elective and urgent surgical lung biopsies. We use open biopsies in patients referred for emergency biopsies. These patients are vulnerable to the risks of increased operative time, reintubation with a double-lumen endotracheal tube, and single lung ventilation. Consequently, the urgency and technique of the procedures are closely related. This approach has been advocated by others. ${ }^{3,4}$

Laterality and site of biopsy were chosen to facilitate the surgical procedure. All patients in this series had diffuse disease; and, with few exceptions, identification of target regions was unnecessary. The lingula and right middle lobe were frequently sampled. These regions have been shown to be satisfactory locations for lung biopsy. ${ }^{5,6}$

Our diagnostic yield was $100 \%$. This compares favorably to reported rates in patients who are immunocompetent of $71 \%$ to $100 \% .{ }^{1,7,8}$ Usual interstitial pneumonia, bronchiolitis, diffuse alveolar damage, honeycomb lung, and pneumonia were our most common diagnoses. Others have reported usual interstitial pneumonia in $27 \%$ of patients who are immunocompetent, bronchiolitis in $18 \%$ to $26 \%$ of patients who are immunocompetent, malignancy in $5 \%$ to $13 \%$ of patients who are immunocompetent, and infection in $0 \%$ to $4 \%$ of patients who are immunocompetent. ${ }^{1,7,8}$

Previous reports site $0 \%$ to $10 \%$ yields for bacterial, fungal, and viral cultures in the elective setting. ${ }^{1,7}$ In patients who are immunocompetent, infection has been found in only $4 \%$ of patients undergoing open lung biopsies. ${ }^{8}$ Although we routinely obtained cultures, we found no clinically significant positive cultures for aerobic bacteria, anaerobic bacteria, or mycobacteria. A single fungal culture was clinically useful and resulted in treatment. The few viral, protozoal, and fungal infections in our series were generally diagnosed pathologically. Thus it appeared that routine cultures were not necessary in our series of predominantly patients who were immunocompetent. 
Significant beneficial therapeutic changes were made in $61 \%$ of our patients as a result of lung biopsy. Previous authors have reported changes in therapy in $46 \%$ to $54 \%$ of all patients undergoing open lung biopsy and in $18 \%$ to $41 \%$ of patients who are immunocompetent. $^{7,8}$ These data are difficult to interpret because definitions vary between authors. Significant beneficial therapeutic changes in our patients consisted of immunosuppression in $87 \%$ of elective cases, $56 \%$ of urgent cases, and $82 \%$ of emergency cases. Significant beneficial therapeutic changes other than immunosuppression occurred in 11 of 69 of the entire group (16\%) in whom therapy after biopsy is known.

Previous reports have documented overall operative mortality rates of $0 \%$ to $60 \% .^{7-9}$ Our overall operative mortality rate was $27 \%$. Operative mortality rates in patients who are immunocompetent has been reported as $0 \%$ to $7 \%$ with elective treatment, $10 \%$ with urgent treatment, and $33 \%$ to $66 \%$ with emergency treatment; again definitions vary., ${ }^{4,-11}$ Our mortality rate depended significantly on urgency (elective treatment, no deaths; urgent treatment, $18 \%$ mortality; emergency treatment, 54\% mortality). In many instances, death resulted from the withdrawal of care in patients who were expected to have minuscule chances for recovery. With the use of univariable analysis, urgency and use of open surgical technique were significantly associated with operative death. With multivariable logistic regression analysis, only urgency remained as significant predictor. We and others have been unable to demonstrate decreased operative death between patients who received new therapies after biopsy and those who did not. ${ }^{4,7,10,12}$

Should lung biopsy be performed? Patients undergoing elective biopsy have no operative deaths, and $60 \%$ obtain significant beneficial therapeutic changes. Patients undergoing urgent biopsy have an $18 \%$ operative mortality rate, but $94 \%$ receive significant beneficial therapeutic changes. There is no significant difference in operative survival between those with significant beneficial therapeutic changes and those without significant beneficial therapeutic changes. To determine whether lung biopsy is advantageous, a comparison between those who undergo biopsy and those who are treated empirically should be performed. Until then, biopsies are appropriate in elective and urgent situations.

Conversely, patients undergoing emergency biopsy have an operative mortality rate of $54 \%$, and only $7 \%$ receive significant beneficial therapeutic changes other than immunosuppression. There is no significant difference in operative survival between those patients receiving significant beneficial therapeutic changes and those patients who do not. A comparison between those patients who undergo biopsy and those patients who are treated empirically should be performed. Until then, these patients should not receive diagnostic surgical procedures if they are in stable condition. They should be treated empirically with immunosuppression without operation if their condition is deteriorating. This approach avoids the risk, cost, and potential liabilities of a surgical procedure that has no apparent or proven benefit to the patient.

\section{REFERENCES}

1. Bensard DD, McIntyre RC Jr, Waring BJ, Simon JS. Comparison of video thoracoscopic lung biopsy to open lung biopsy in the diagnosis of interstitial lung disease. Chest 1993;103:765-70.

2. Carnochan FM, Walker WS, Cameron EW. Efficacy of video assisted thoracoscopic lung biopsy: a historical comparison with open lung biopsy. Thorax 1994;49:361-3.

3. Ferguson MK. Thoracoscopy for diagnosis of diffuse lung disease. Ann Thorac Surg 1993;56:694-6.

4. Canver CC, Mentzer RM Jr. The role of open lung biopsy in early and late survival of ventilator-dependent patients with diffuse idiopathic lung disease. J Cardiovasc Surg 1994;35:151-5.

5. Miller RR, Nelems B, Muller NL, Evans KG, Ostrow DN Lingular and right middle lobe biopsy in the assessment of diffuse lung disease. Ann Thorac Surg 1987;44:269-73.

6. Wetstein L. Sensitivity and specificity of lingular segmental biopsies of the lung. Chest 1986;90:383-6.

7. Walker WA, Cole FH Jr, Khandekar A, Mahfood SS, Watson DC. Does open lung biopsy affect treatment in patients with diffuse pulmonary infiltrates? J Thorac Cardiovasc Surg 1989;97:53440.

8. Kramer MR, Berkman N, Mintz B, Godfrey S, Saute M, Amir G. The role of open lung biopsy in the management and outcome of patients with diffuse lung disease. Ann Thorac Surg 1998;65:198202.

9. LoCicero J III. Does every patient with enigmatic lung disease deserve a lung biopsy? Chest 1994;106:706-8.

10. Bove P, Ranger W, Pursel S, Glover J, Bove K, Bendick P. Evaluation of outcome following open lung biopsy. Am Surg 1994;60:564-70.

11. Lachapelle KJ, Morin JE. Benefit of open lung biopsy in patients with respiratory failure. Can J Surg 1995;38:316-21.

12. Warner DO, Warner MA, Divertie MB. Open lung biopsy in patients with diffuse pulmonary infiltrates and acute respiratory failure. Am Rev Respir Dis 1988;137:90-4. 